FORUM: Climate change

Flowering in the greenhouse

Predicting plant responses to increasing temperatures is integral to assessing the global impact of climate change. But the authors of a comparative study assert that warming experiments may not accurately reflect observational data. Climate and ecosystem scientists discuss how impact prediction should proceed. SEE LETTER P.494

\section{THE PAPER IN BRIEF}

- Seasonal plant flowering and leafing have occurred earlier in recent decades, almost certainly as a result of higher average temperatures.

- Artificial warming experiments are designed to replicate changed climatic conditions in a controlled environment, allowing direct assessment of

\section{Consider all data}

\section{THIS RUTISHAUSER \& RETO STÖCKLI}

Dhenology, the science of seasonally recurring life-cycle stages in plants and animals, has become an important aspect of climate science. Anthropogenic climate change is leading to anomalous conditions, including warming trends that strongly correlate with changing plant flowering and leafing dates. In turn, these unusual phenological manifestations may lead to anomalous bird migration and to plant pollination patterns that could desynchronize entire food chains and have socio-economic impacts that remain poorly defined. Observational studies are needed to document and understand these changes, and such studies are essential to constrain models used to estimate the effects of projected future warming on Earth's carbon and water cycles.

One open question is whether temperature sensitivities derived from past phenological observations will remain valid in a future, warmer climate. To answer this question, biologists typically rely on plant temperature sensitivities (defined as the number of days' change in flowering or leafing date per degree of temperature change) determined from controlled, plot-scale field experiments in which vegetation is warmed artificially. Wolkovich and her interdisciplinary team ${ }^{1}$ compared the sensitivities ascertained from long-term observational records with results from

the effects on plants

- Wolkovich et al. ${ }^{1}$ (page 494) compare existing data on the responses of 1,634 plant species, across four continents, to observed and experimental warming.

- They find that observed plant flowering and leafing dates have occurred significantly earlier than the changes calculated from artificial warming experiments.

warming experiments. They compiled data on temperature sensitivities of 1,634 plant species across multiple latitudes and temporal scales, which dated as far back as the nineteenth century. They found that flowering and leafing sensitivities obtained from warming experiments systematically underpredict those from long-term observational records by a factor of 8.5 and 4.0 , respectively. Their analysis shows that these mismatches do not depend on latitude and that there is little evidence that experimental design influenced the degree of underprediction.

It is not surprising that plant responses to warming-only field experiments are lower than to real-world

\section{"Wolkovich and colleagues' call for} ecosystem-model re-evaluation must be taken seriously." variance occurring over many decades. Temperature changes are not independent of other environmental changes - warm spring seasons, for example, are often accompanied by more sunshine, drier soils and shortened snow duration. All of these factors influence plant growth and phase-change events, such as budding and flowering ${ }^{2}$. Moreover, temperature sensitivities are neither constant in time nor fixed within a species, and can vary by between 3 and 6 days per degree when data recorded over centuries are considered ${ }^{3}$.

The difficulty in replicating these factors experimentally means that Wolkovich and colleagues' call for ecosystem-model re-evaluation must be taken seriously. Current vegetation models predict either a delayed or lengthened plant growing season, either of which will have adverse effects on the terrestrial carbon uptake. But recent studies have revealed substantial deficiencies in these models. For example, exceptionally warm winter temperatures can disrupt a plant's requirements for chilling, and thus, apparently paradoxically, delay leafing and flowering ${ }^{5}$. Phenology is much more than a linear correlate to annual mean air temperatures - for a model to make realistic phenological predictions, it must combine the effects of, at least, temperature, light and moisture ${ }^{6}$.

The real and potential impacts of phenological change on natural ecosystems attract attention from scientists and the public alike, as, for example, in the interview ${ }^{7}$, 'What's the impact of early blooms?', which was broadcast in March on the US National Public Radio programme All Things Considered, and the burgeoning number of 'citizen science' projects, in which individuals provide researchers with observational field data (see, for example, refs 8-10). The prediction discrepancies revealed by Wolkovich and colleagues can be resolved only by further analyses of long-term phenological observations in combination with climate and ecosystem data, backed by model-based and experimental results. The key message of this study is that we must consider, and obtain, more observational data to better understand the processes of the global 'warming experiment' that is ongoing on our planet - one that is taking place daily, without control plots and on the most relevant scale. We must put all these data to use.

This Rutishauser is at the Oeschger Centre for Climate Change Research, the Institute of Geography, University of Bern, and the Commission for Phenology and Seasonality, Swiss Academies of Sciences SCNAT, CH-3012 Bern, Switzerland. Reto Stöckli is at the Federal Office of Meteorology and Climatology MeteoSwiss, CH-8044 Zurich, Switzerland. e-mails:rutis@giub.unibe.ch; reto.stoeckli@meteoswiss.ch 


\section{Insight from integration}

\section{JOHN HARTE \& LARA KUEPPERS}

$\mathrm{A}$ nthropogenic climate change, a massive uncontrolled planetary disruption, is under way, and a predictive understanding of how it will affect ecosystems and how these ecosystem responses will feed back to the climate is badly needed. Meta-analyses, such as that presented by Wolkovich et al. ${ }^{1}$, have the potential to augment current ecological understanding. But when such analyses fail to explicitly address methodological differences between data sources that are crucial for interpreting results, their conclusions can be misleading. Here, we urge caution in the use of meta-analyses to assess different approaches to predicting ecosystem responses to climate change, and we describe how integrating multiple investigative methods can better advance understanding.

To predict ecosystem responses, scientists can observe correlations between climate and ecosystem properties, and can conduct controlled experimental manipulations of climate. However, both methods have limitations as well as strengths - it is difficult, for example, to identify causal mechanisms from correlative observations, and controlled experiments on small spatial scales cannot reflect all aspects of larger environments. Predictions of ecosystem responses should strive to make use of information from differing approaches in a way that considers the strengths and drawbacks of each.

Simply comparing results from many different experimental and correlative studies, as Wolkovich and colleagues attempted, can obscure nuances of methodology and analysis that are essential for making accurate predictions. Alternatively, integrating observational and experimental methods within a single study ${ }^{11}$ can simultaneously provide insight into issues that are obscured by meta-analyses and the mechanisms driving ecosystem responses. For example, we have studied the sensitivity of subalpine meadow plants to climate using two types of experimental climate change (yearround heating and snow removal) and two types of natural climate change (interannual variability and change along an elevation gradient). We found ${ }^{12}$ that the timing of flowering, and many other ecological responses, are remarkably similarly sensitive to each of these four sources of climate change, provided that snow-melt date is used as an explanatory variable, rather than mean annual temperature, as used by Wolkovich and colleagues.

The type of experimental method used for such studies does matter, though. Had we used passive heating methods, such as opentop chambers (Fig. 1) deployed only during the growing season, we might have increased

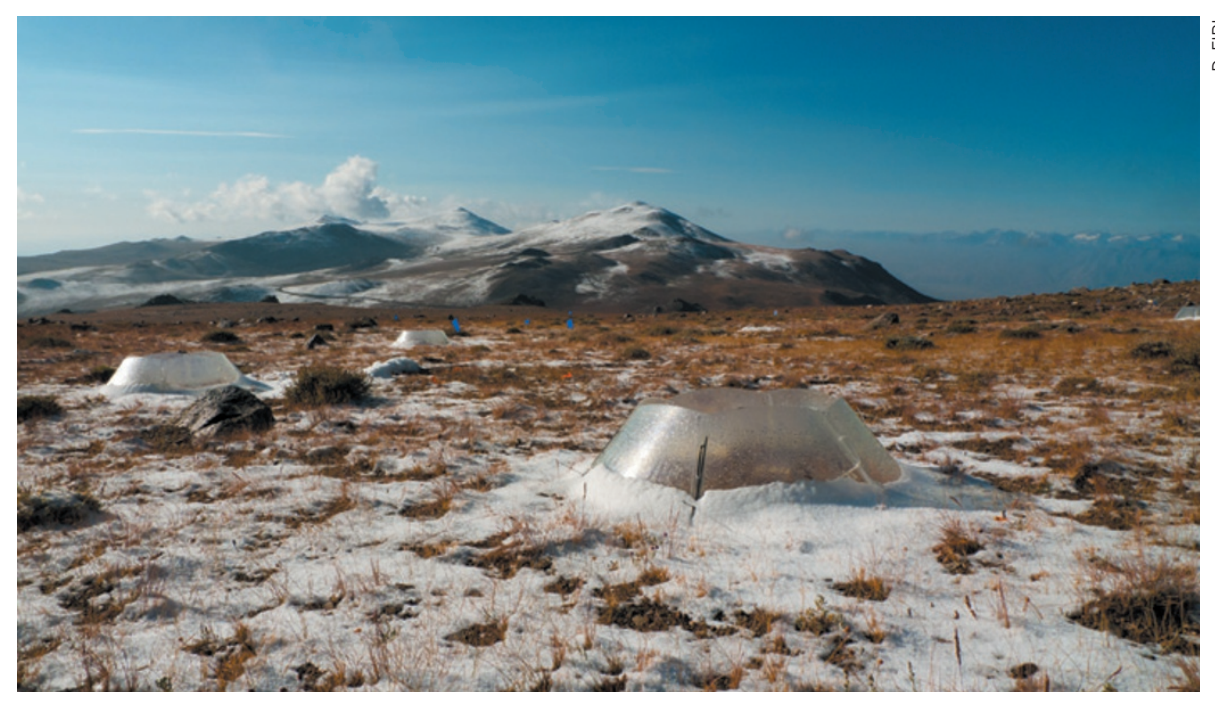

Figure 1 | Early bloomers. Artificial warming experiments, such as that shown here in the White Mountains of California, in which plants growing in open-top warming chambers are compared with adjacent plants growing in normal conditions, can be used to study how plant flowering and leafing times alter in different temperature conditions, and thereby help to predict how plants will respond to climate change.

the mean annual temperature, but our experimental heating would not have advanced snow melt and we would not have observed a strong phenological response. Thus, meta-analyses should carefully consider both the appropriate explanatory variables and methodological differences across experimental studies. Explanatory variables are likely to differ from one type of habitat to another, and from one type of response variable to

"Explanatory
variables are
likely to differ
from one type
of habitat to
another."
another. In lowland tropical regions, where snow is not a factor, there is a great need for integrative methods to determine which variables drive plant and animal, as well as biogeochemical, responses to climate change.

Our observation that phenology responds to changing snow-melt date consistently across different observational and experimental methods might suggest that researchers could dispense with expensive warming experiments and rely solely on cheaper, easier correlative studies. But that would be a mistake. Diverse ecosystem responses play out over a range of timescales and require persistent multi-year manipulations to uncover causal mechanisms ${ }^{13}$. For example, the change in soil carbon levels that occurs over time in response to persistent shifts in plant-species composition is not easily captured by interannual climate variability, but we recorded it in our controlled 21-year warming experiment ${ }^{14}$. Furthermore, predicting climate impacts on species turnover, selection and adaptation may require integration of yet other types of observation and experiment, such as the use of data from the fossil record and reciprocal transplant experiments across climate gradients.
These examples demonstrate how controlled warming experiments, particularly those that proceed for longer than the typical research-funding cycle of 3-5 years, can provide a means of understanding both patterns in ecosystem responses and the multiple mechanisms that govern these changes. Such experiments will allow identification and manipulation of explanatory variables and, if they are performed in combination with observational studies that take advantage of climate variability in space and time, will guide our quest to predict the future of ecosystems under global change.

John Harte is at the University of California, Berkeley, Berkeley, California 94720, USA.

Lara Kueppers is at the University of California, Merced, Merced, California 95343, USA.

e-mails: jharte@berkeley.edu;

lkueppers@ucmerced.edu

1. Wolkovich, E. M. et al. Nature 485, 494-497 (2012)

2. Körner, C. in Plant Growth and Climate Change (eds Morison, J. I. L. \& Morecroft, M. D.) 48-69 (Blackwell, 2006).

3. Rutishauser, T., Luterbacher, J., Defila, C., Frank, D. \& Wanner, H. Geophys. Res. Lett. 35, L05703 (2008).

4. Richardson, A. D. et al. Glob. Change Biol. 18, 566-584 (2012).

5. Vitasse, Y. C. et al. Agric. Forest Meteorol. 151, 969-980 (2011).

6. Stöckli, R., Rutishauser, T., Baker, I., Liniger, M. A \& Denning, A. S. J. Geophys. Res. 116, G03020 (2011).

7. www.npr.org/2012/03/15/148698272/whats-theimpact-of-early-blooms

8. www.phaeno.ethz.ch/globe

9. www.blommar.nu

10. naturescalendar.org.uk

11.Dunne, J. A., Saleska, S. R., Fischer, M. L. \& Harte, J. Ecology 85, 904-916 (2004)

12.Dunne, J. A., Harte, J. \& Taylor, K. J. Ecol. Monogr. 73, 69-86 (2002)

13.Smith, M. D., Knapp, A. K. \& Collins, S. L. Ecology 90, 3279-3289 (2009)

14.Saleska, S. R. et al. Glob. Biogeochem. Cycles 16, 1055 (2002). 